\title{
Decentralised Control of DC Microgrid Based on Virtual Admittance to Enhance DC Voltage and Grid Frequency Support
}

\author{
Syiska Yana \\ Institute for Energy and \\ Environment \\ University of Strathclyde \\ Glasgow, UK \\ syiska.yana@strath.ac.uk
}

\author{
Anthony Florida James \\ Institute for Energy and \\ Environment \\ University of Strathclyde \\ Glasgow, UK \\ anthony.florida-james@strath.ac.uk
}

\author{
Abdullah Emhemed \\ Institute for Energy and \\ Environment \\ University of Strathclyde \\ Glasgow, UK \\ abdullah.emhemed@strath.ac.uk
}

\author{
Graeme Burt \\ Institute for Energy and \\ Environment \\ University of Strathclyde \\ Glasgow, UK \\ graeme.burt@strath.ac.uk
}

\begin{abstract}
DC microgrid technology has become one of key smart grid research topics in recent years. In comparison to $\mathrm{AC}$ microgrids, DC microgrids are more manageable to operate in grid-connected and islanded modes, and also offering improved efficiency and better controllability. Services, such as voltages and AC system frequency support can also be potentially provided by optimally controlling the DC microgrids converter interfaces and their local distributed energy resources. These will require a good understanding of the dynamic interactions between the DC microgrid and the host AC system, and implementation of the appropriate control strategies. This paper investigates the dynamic resilience of a DC microgrid connected to an AC system under different frequency and voltage disturbances. A decentralised droop control strategy within the DC microgrid is used for fast active power control and wider system frequency support. A virtual admittance method is also utilised to enhance the local DC microgrid voltages during the AC frequency events and DC fault test scenarios. The effectiveness of the control strategy is evaluated by simulation studies in MATLAB/Simulink.
\end{abstract}

Keywords-DC microgrids, grid frequency support, DC voltage enhancement, decentralised control strategy

\section{INTRODUCTION}

The increasing development and integration of Distributed Energy Resources (DERs) in recent years give positive contributions to the bulk power system, by reducing the reliance on fossil fuel power plants and improving the sustainability of power resources. This integration has also caused the transformation of conventional power networks, from passive systems into active systems. This transformation is influenced by the location of DERs which are often located at the distribution level. Furthermore, the massive integration of DERs also causes several issues in the power system related to protection, stability, and power quality [1]. To overcome these issues, microgrid's architectures have been widely recommended to integrate and coordinate DERs units, such us photovoltaic (PV) generators, wind turbines (WTs), fuel cells (FCs), and energy storage systems into distribution networks [2]. In general, there are two types of microgrids; $\mathrm{AC}$ microgrid (AC-MG) and DC microgrid (DC-MG). DC-MG will be explored in this study.
Many studies show that DC-MGs have more advantages than AC-MGs [2]-[5]. Various DERs, such as PV and energy storage systems, generate DC power. Therefore, these DERs are more efficiently connected to the DC bus by reducing the conversion stages and associated losses. Also, with no skin effect phenomenon in DC cables, DERs can deliver more real power. Additionally, the synchronisation process to integrate multiple DERs within the DC-MG is not an issue, thus reducing the complexity of the system operation.

As a controllable system, DC-MGs enable operation in two operating modes; grid-connected and islanded mode [4]. For grid-connected mode, the DC-MG is connected to the main grid through a bidirectional DC-AC converter such as two-level voltage source converter (VSC). In this grid connected operating mode, the VSC plays an important role in the power exchange between DC-MG and the main grid and can also provide some ancillary services to the wider system, such as voltage controls and wider system frequency support. So far, there have been few studies into frequency support services provided by DC-MGs [6]. Various research has mostly focused on control strategies for addressing local parameters of DC-MGs such as power sharing, and local voltage stability within the microgrid boundaries [7]-[9]. Furthermore, the resources within the DCMG are electrically decoupled from the main grid and therefore do not contribute to system inertia. With the penetration of inverter interfaced DER increasing, and the synchronous plant being closed or mothballed, the system inertia is consequently decreasing, which leads to a higher rate of change of frequency (RoCoF) [10], [11]. Over and above, the DERs within the DC$\mathrm{MG}$ are decoupled from the $\mathrm{AC}$ grid, resulting in further regional inertia reduction. Therefore, it is important to understand to what extent wider system frequency disturbances will impact the dynamic performance of the DC-MG and the control measures that are required to mitigate any resultant impact within and without the microgrid boundaries.

In response to this, this paper investigates the dynamic performance a DC-MG connected to an AC system with reduced inertia. The focus of the paper evaluates the capability of DCMGs to provide wider AC system frequency support and at the same time improve the local performance of the DC voltage within the microgrid. The paper applies a decentralised control 
strategy through conventional frequency-active power (F-P) droop control and a virtual admittance to improve the resilience of the DC-MG and the host AC systems during frequency event and DC voltage dips fault conditions. Two case studies are conducted to evaluate the performance of DC-MG and control strategies. The first case study investigates the DC-MG as a provider of grid frequency support and the second case study investigates DC voltage enhancement by virtual admittance.

The rest of the paper is organised as follows: Section II presents a general overview of grid frequency regulation. Section III describes modelling of the DC-MG and VSC grid frequency support control strategy, including virtual admittance based droop control at the battery. Section IV presents the simulation results and discussion. Section V provides the conclusions of the paper.

\section{AN OVERVIEW OF GRID FREQUENCY RESPONSE}

In GB, the system operator National Grid is responsible for ensuring frequency stability. There are three types of frequency regulation services which are primary and secondary frequency response [12]. Fig.1 shows system frequency evolution after an event [13]. According to National Grid Mandatory Frequency Response in [14], to participate as grid frequency support provider, the provider must have capacity $\geq 1 \mathrm{MW}$ and able to detect the grid frequency deviation within $500 \mathrm{~ms}$.

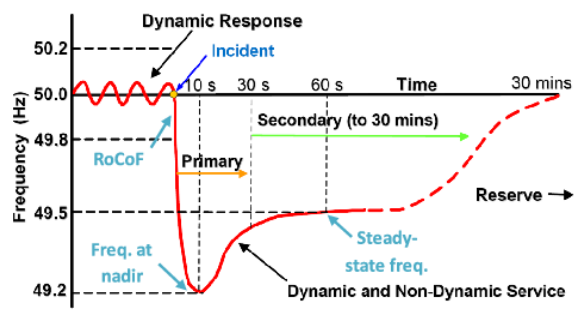

Fig. 1. System frequency evolution after an event [13]

Based on the National Grid requirements, the DC-MG must be able to provide a minimum of $1 \mathrm{MW}$ to participate in grid frequency support. Even though the DC-MG can meet the VSC capacity requirement for grid frequency support, there is a problem related to the high proportion of renewable energy resources, such as PV generation and battery energy storage devices, within the microgrid (Fig.2). As mentioned in the previous section, the VSC interfaced resources cause a reduction of inertia in the host power system and impact the initial RoCoF when a disturbance occurs [10], [11]. There have been many studies conducted into grid frequency support using VSC control strategies for various DERs, such as PV, WT, energy storage devices, and smart loads [15]-[20]. However, only a few studies are concerned with the DC-MG as grid frequency support [6]. The main challenge of the DC-MG when providing frequency support is how this system, and its control strategy, can emulate the required inertia to effectively support the grid frequency. The following section will explain the DC-MG test network and control strategy that is implemented for this study.

\section{MODELLING OF DC MICROGRID AND GRID FREQUENCY SUPPORT CONTROL STRATEGY}

This section is comprised of two sub-sections. The first subsection presents the DC-MG test network. The second sub- section explains the VSC and DC voltage virtual admittance based control strategies that enabled DC-MG grid frequency support and improved DC fault ride through.

\section{A. Test Network}

In this study, the DC-MG integrates PV generation, battery energy storage, and load. These are all connected to the twolevel VSC and AC system in the low voltage (LV) side, as shown in Fig.2. The PV generator, energy storage device and load are integrated into the $750 \mathrm{~V}_{\mathrm{DC}}$ bus. This voltage level enables direct connection to $400 \mathrm{~V}_{\mathrm{AC}}$, and a higher power transmission capacity [21]-[23]. The DC-MG is connected through $11 / 0.4 \mathrm{kV}$ transformer and 1 MVA VSC. The representative host system is supplied by a 350 MVA synchronous machine with the IEEE Type 1 excitation system, as well as a steam governor and turbine model. Further system parameters are shown in Table 1.

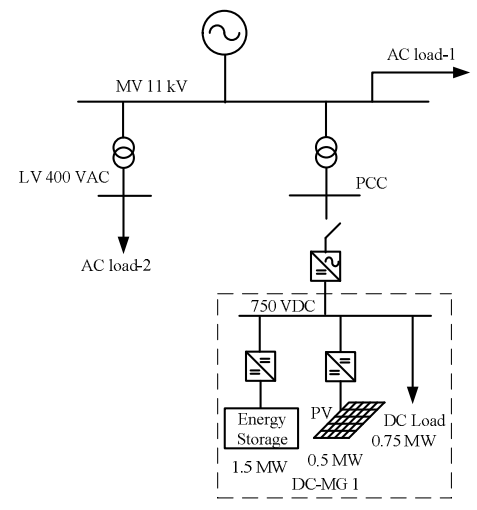

Fig. 2. DC-MG test network

TABLE I. SYSTEM PARAMETERS

\begin{tabular}{ll}
\hline \hline & Parameters \\
\hline \hline Synchronous machine & $350 \mathrm{MVA}, 11 \mathrm{kV}, 50 \mathrm{~Hz}$ \\
Transformers & $5 \mathrm{MVA}, 11 / 0.4 \mathrm{kV}, 50 \mathrm{~Hz}$ \\
AC-load 1 & $250 \mathrm{MW}$ \\
AC-load 2 & $0.24 \mathrm{MW}, 0.104 \mathrm{MVAR}$ \\
VSC rating & $1 \mathrm{MW}$ \\
\hline \hline
\end{tabular}

Due to the relatively fast dynamic response requirements of the studies, the PV unit is set to generate $0.6 \mathrm{pu}$ of the maximum rated power $(500 \mathrm{~kW})$, without temperature and irradiance variations [24]. Furthermore, $600 \mathrm{~V}_{\mathrm{DC}}$ Lithium-Ion battery is utilised with an initial State of Charge (SoC) of $80 \%$. The battery is connected to the DC bus through a bidirectional DCDC buck-boost converter.

\section{B. Control Strategy}

The VSC control strategy based on conventional droop control to provide power exchange between the AC system and DC-MG during normal operation and the frequency event is utilised. Three operation modes are utilised to create the initial condition before the event to show the flexibility of DC-MG and described as follows:

\section{a) Self-consumption mode}

In this mode, the load in the DC-MG is fully supplied by DERs within the microgrid and when the frequency 
event occurred in the AC side, the DC-MG injected or absorbed the power to and from the main grid.

\section{b) Import mode}

The DC-MG load is supplied by the synchronous machine from $\mathrm{AC}$ host system and the resources within the DC-MG.

\section{c) Export mode}

The load in DC-MG is fully supplied by the resources within the microgrid and spare capacity is injected to the AC system.

The VSC controller consists of an outer power control loop and inner current control loop. The active and reactive power set-points are determined by the F-P droop curve with a deadband of $50 \pm 0.015 \mathrm{~Hz}$.

On the DC side, V-I droop control is implemented for the DC-DC buck-boost converter, which interfaced the battery to the DC bus. The block diagram of the controllers is shown in Fig.3.

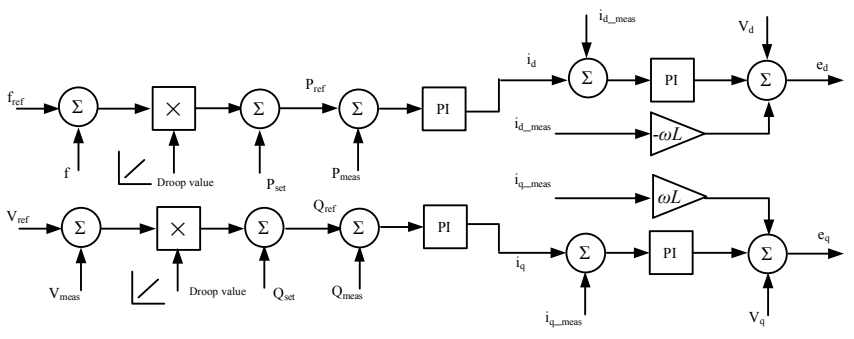

(a)

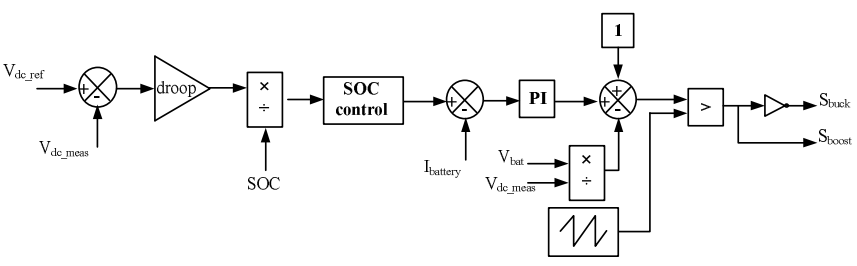

(b)

Fig. 3. (a) VSC controller, (b) DC-DC buck-boost controller

To improve the droop control performance and enhance the DC voltage, a virtual admittance is utilised as part of the V-I droop control. The virtual admittance reduces the offset error of the proportional droop calculation. Then, the current control can be actuated based on the DC voltage, injecting or absorbing more current when the load or generation increases or decreases. Fig. 4 shows the representation of virtual admittance in DC-MG.

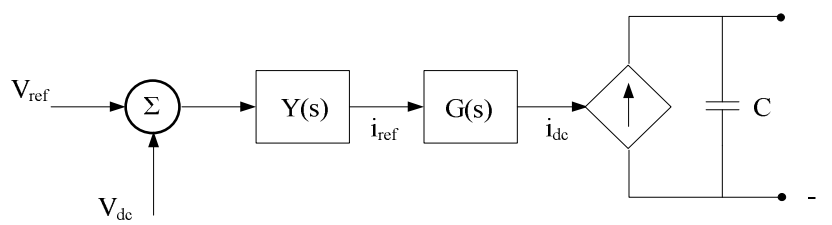

Fig. 4. Representation of virtual admittance in DC-MG [25]
Virtual admittance is annotated as $\mathrm{Y}(\mathrm{s})$. The controller annotated as $\mathrm{G}(\mathrm{s})$ is shown with the current source and is representative of DC-DC buck-boost converter. The relation between Y(s) and G(s) is stated in equation (1) [25]. From the equation (1) and Fig.4, $\mathrm{V}_{\text {ref }}$ is the voltage set point and $\mathrm{i}_{\text {ref }}$ is the current reference for the buck-boost converter. Parameters for the controllers are shown in Table II.

$$
Y(s)=\frac{i_{r e f}(s)}{V_{r e f}(s)-V_{d c}(s)}
$$

TABLE II. CONTROLLER PARAMETERS

\begin{tabular}{ll}
\hline \hline \multicolumn{2}{c}{ Controller Parameters } \\
\hline \hline VSC controller parameters: \\
Switching frequency & $2 \mathrm{kHz}$ \\
Desired bandwidth & 100.53 \\
Rf & $1.2 \mathrm{~m} \Omega$ \\
Lf & $1.6 \mathrm{mH}$ \\
ki1, ki2 & 38,25 \\
kp1, kp2 & $4,0.05$ \\
DC-DC buck-boost controller parameters: & \\
Switching frequency & $1 \mathrm{kHz}$ \\
L & $2 \mathrm{mH}$ \\
Droop gain & 12 \\
Proportional gain & 12 \\
Integral gain & 25 \\
Virtual admittance gain & 3 \\
\hline \hline
\end{tabular}

\section{SimUlation RESUltS AND DISCUSSION}

MATLAB/Simulink software is utilised to verify the DCMG test network and control strategy for wider system frequency support and DC voltage enhancement, by conducting two case studies as follows:

a) DC-MG for grid frequency support

This case study is conducted to evaluate the capability of the DC-MG to provide wider system frequency. In this scenario, the frequency event of the system is simulated with and without the DC-MG. Three different pre-event operating modes (as explained in Section. III. $B$ ) are conducted to show the dynamic behaviour and flexibility of the DC-MG.

b) Virtual admittance for DC voltage control

This case study is conducted to show the influence of virtual admittance and its ability to improve the DC voltage during the event, and also during fault conditions. There are two fault types investigated in this study. First, pole-to-ground fault and second, pole-to-pole fault.

The following sub-sections will provide more detail of the two case studies, including the simulation results and discussion.

\section{A. DC-MG for grid frequency support}

In this scenario, the frequency event of the system is simulated with and without the DC-MG. A load step changer is connected to create the frequency disturbance in the system. The simulation is conducted by implementing pre-event three 
operating modes. Fig.5 shows the simulation results which comprise of frequency response, VSC power and the DC voltage profile.

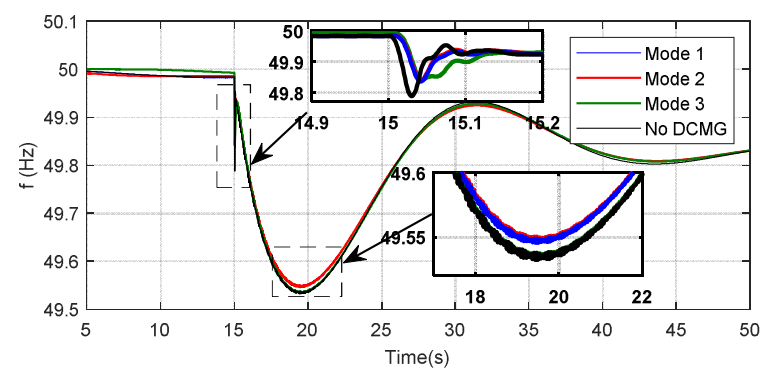

(a)

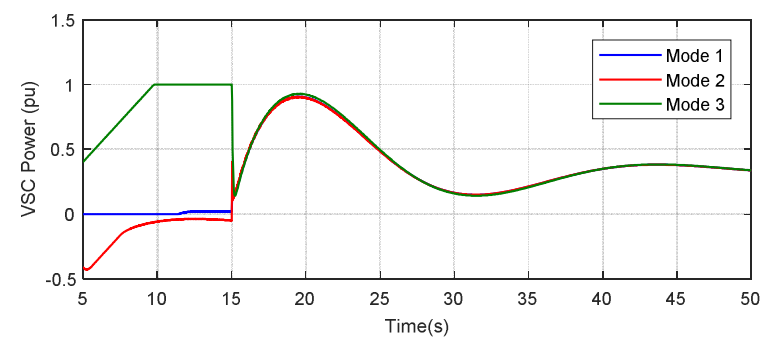

(b)

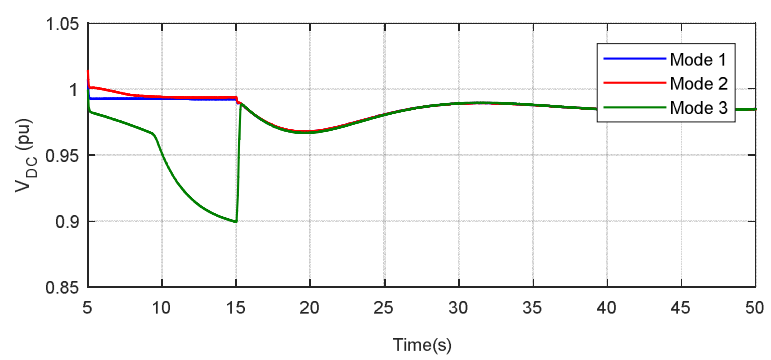

(c)

Fig. 5. DC-MG response (a) frequency, (b) VSC power, and (c) DC voltage

Fig 5(a) shows the comparison of the frequency response of the test network with and without the DC-MG. The effect of the three pre-event operating modes can be clearly seen in the zoom sections on the graph. From the simulation results shown in Fig.5 (a), it can be seen that DC-MG enables to enhance the frequency of the AC system. The best frequency response is provided following the DC-MG using Mode 2. This is due to a 'demand response' effect, followed rapidly by the VSC power injection to the system. This provides a better frequency response when compared to the other two modes. For example, from Fig. 5(a), it can be seen that there is an improvement not only for the frequency nadir but also the RoCoF is less for all modes. The fastest response is provided by Mode 2. The improvement of the frequency nadir for all modes is not too significant because, when compared to the capacity of the AC system, it is a relatively small amount of power. The DC-MG in this study can therefore be categorised as a small system [12]. However, if many VSCs for DC-MG integration employed the control used in this study, there would be a greater provision of frequency support throughout the system.

Furthermore, in Fig. 5(b) the power exchange between the DC-MG and the AC system during the frequency event based on three operating modes are also presented. Before the frequency event occurred at $15 \mathrm{~s}$, it can be seen that the DC-MG absorbs and injects the power in Mode 2 and 3, respectively. Then, during the frequency event, the DC-MG injects the power to the AC system for all operating modes.

Moreover, Fig. 5(c) shows the DC voltage profile for all modes during the frequency event. From Fig. 5(c), it can be seen that the worst impact for the DC voltage before the event occurred whilst the DC-MG utilised Mode 3. This is due to the magnitude of power being exported into the grid pre event.

Additionally, the impact of virtual admittance for grid frequency support is also investigated in this study. However, it can be seen in Fig. 6, there is no significant impact of virtual admittance to enhance the system frequency. The following section will present the impact of virtual admittance to enhance the DC voltage controller.

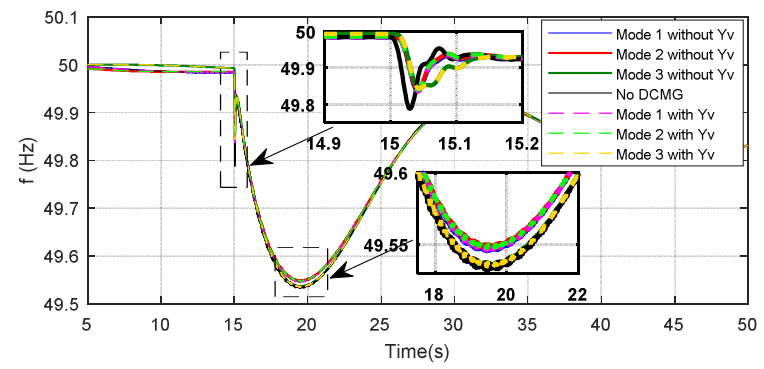

Fig. 6. Frequency response by using virtual admittance

\section{B. Virtual admittance to enhance DC voltage control}

In this scenario, the ability of virtual admittance to enhance $\mathrm{V}_{\mathrm{DC}}$ control is investigated. As shown in Fig.5(c), the initial condition of pre-event and frequency event in the main grid impact the DC-MG voltage. When the frequency event occurs at $15 \mathrm{~s}, \mathrm{~V}_{\mathrm{DC}}$ deviates slightly, just remaining in the range of $\mathrm{DC}$ voltage normal operation standard $\pm 10 \%[21]$. To reduce the DC voltage deviation in the DC-MG, virtual admittance is implemented in this study. Fig. 7 shows the performance of the controller with virtual admittance to improve the voltage in the DC side. From Fig.7, it can be seen that virtual admittance improves the DC voltage during the frequency event. However, there is no significant improvement for the DC voltage before the event. It seem that virtual admittance only effective to improve the voltage which is declined not less than $0.9 \mathrm{pu}$.

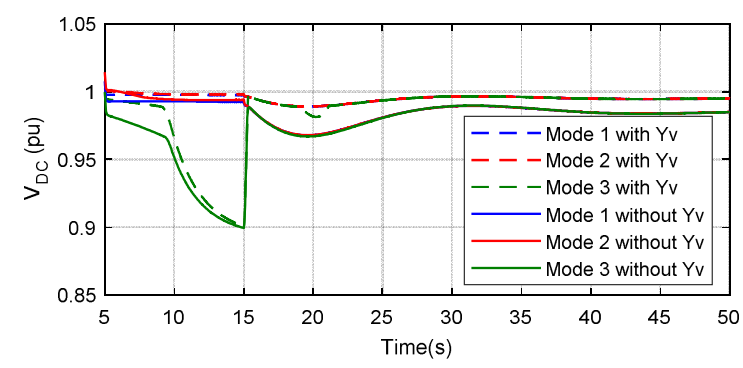

Fig. 7. Comparison of $\mathrm{V}_{\mathrm{DC}}$ during the frequency event

Further investigation is also conducted to test the impact of virtual admittance during DC fault condition (pole-to-ground fault and pole-to-pole fault). The distance of the fault location 
for both fault conditions is neglected as this study is not focused on the protection issue. The fault duration for pole-to-ground fault is set to $5 \mathrm{~ms}$ with fault resistance of $0.001 \mathrm{Ohm}$. For poleto-ground fault, the simulation only conducted for one fault resistance because the result for the variations of the resistance is not too significantly different, when compared to pole-to-pole fault test. There are two DC-MG operating conditions used for this fault behaviour investigation. First, faults are introduced when the DC-MG had no load, and second when the DC-MG load is connected. Both of these conditions are tested with and without virtual admittance. For the first condition, virtual admittance enables the DC voltage to reduce the steady state error and stay close to $1 \mathrm{pu}$ as seen in Fig. 8(a). Then, for the second condition shown in Fig. 8(b), virtual admittance enables to boost the DC voltage up to $1 \mathrm{pu}$, thus enabling greater power transfer on the DC link. For this fault condition, the DC voltage does not drop to zero due to the voltage potential difference of the battery.

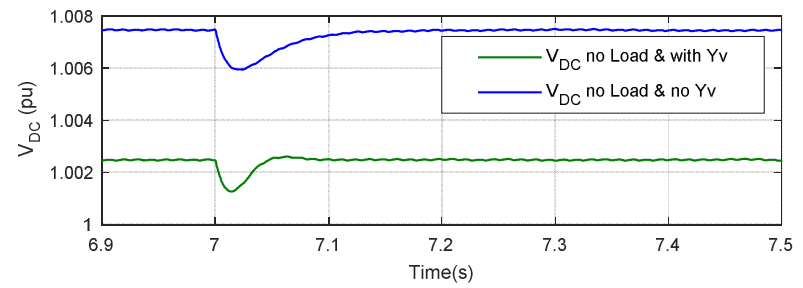

(a)

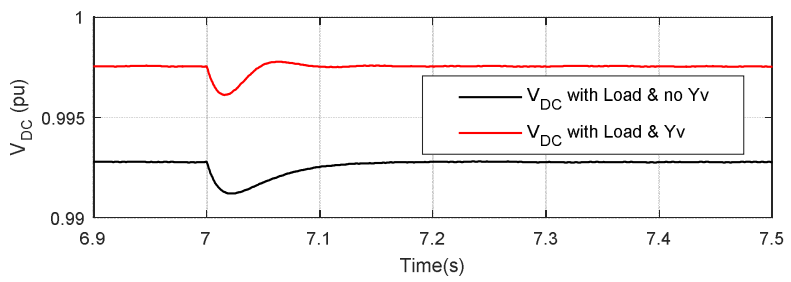

(b)

Fig. 8. Comparison of $\mathrm{V}_{\mathrm{DC}}$ for pole-to-ground fault test

For pole-to-pole fault scenario, the fault duration is set to $0.01 \mathrm{~s}$ for different fault resistance $(0.001,0.01,0.1$ and $1 \mathrm{Ohm})$. Fig. 9 shows the comparison of the impact virtual admittance on the DC voltage during the faults. Similar to the pole-to-ground fault, the DC voltage did not drop to zero during the fault event. Fig. 9(a) shows the voltage profile during the fault occur with fault resistance $0.001 \mathrm{Ohm}$. It can be seen there is no significant difference between the condition with and without virtual admittance. During the fault condition, the voltage drops to $0.6 \mathrm{pu}$ and the control strategy is unable to restore the voltage. However, for this condition, the DC voltage still remained close to $1 \mathrm{pu}$ for steady state conditions. It can be concluded that the control strategy and virtual admittance only enhances voltage which is decreased not less than or above $0.9 \mathrm{pu}$, as can be seen in frequency event case for Mode 3 in Fig. 7. Stability assessment of the controller in future work can provide further insight into the limits of the controller capabilities. The empirical analysis above is also supported by the evidence from Fig. 9(b)-(d). For fault resistance $0.01 \mathrm{Ohm}$, the voltage decreased to $0.84 \mathrm{pu}$ and the virtual admittance only impacted the voltage pre and post event. Conditions in Fig. 9(a) and (b) are in contrast to Fig. 9(c) and (d). From the Fig. 9(c) and (d), during the fault, the voltage did not drop below $0.9 \mathrm{pu}$. Therefore, the control strategy and virtual admittance are still enable to enhance the voltage.

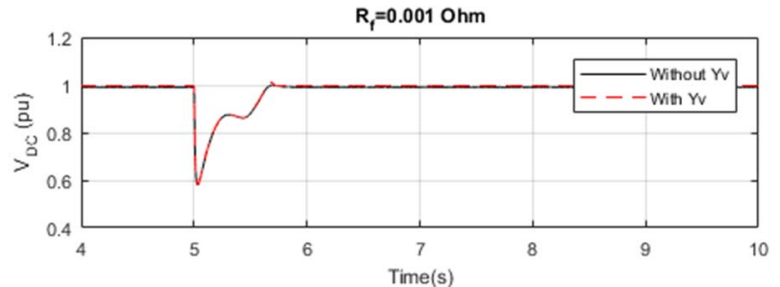

(a)

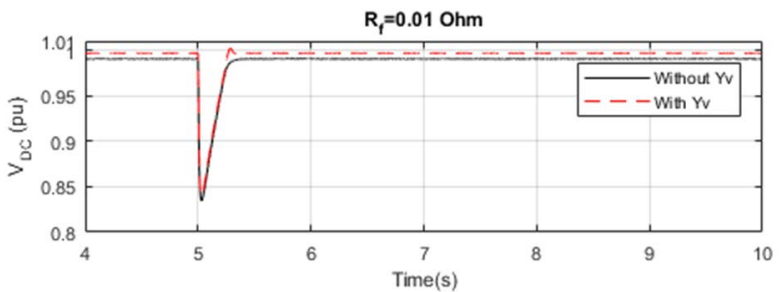

(b)

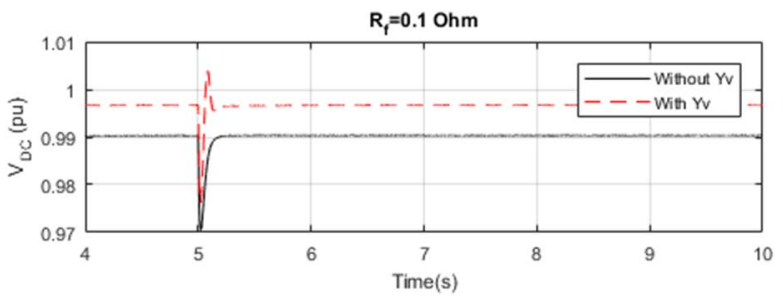

(c)

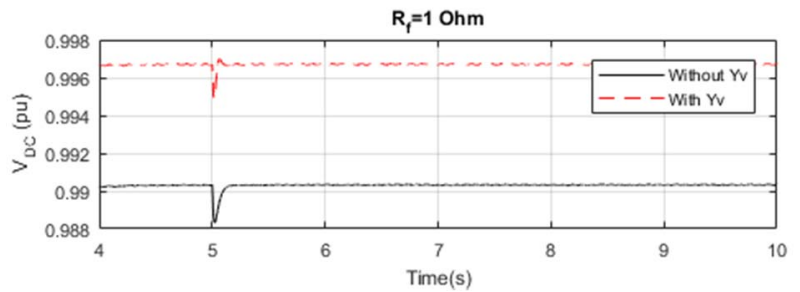

(d)

Fig. 9. Comparison of $\mathrm{V}_{\mathrm{DC}}$ for pole-to-pole fault test

\section{CONCLUSIONS}

In this study, DC-MG control strategy based on conventional F-P droop and $\mathrm{V}_{\mathrm{DC}}-\mathrm{I}$ droop with virtual admittance is proposed to enhance the AC system frequency support capability and DC$M G$ voltage control. Two scenarios have been conducted to test the performance of the DC-MG test network and control strategy. The first scenario focused on DC-MG as grid frequency support and second scenario discussed the DC voltage control. Then, the impact of virtual admittance to improve the DC voltage is also investigated during the frequency event and fault test. Simulation results show the effectiveness of the control strategy to enhance system frequency, power exchange, and DC voltage regulation. However, the control strategy and virtual admittance only enable to enhance the DC voltage above $0.9 \mathrm{pu}$. Therefore, further investigation and development for robust control strategy is needed. Future work will also develop further study to investigate the control strategy for multiple DC- 
MGs with various resources and operating modes for grid frequency support and DC-MG stability.

\section{ACKNOWLEDGMENT}

The authors would like to acknowledge support from the LPDP (Indonesia Endowment Fund for Education), Indonesia Ministry of Research, Technology and Higher Education (RISTEK DIKTI) through the BUDI-LN scheme and Universitas Sumatera Utara. This study is also supported by EPSRC CDT in Future Power Networks and Smart Grids.

\section{REFERENCES}

[1] NERC, "Distributed Energy Resources Connection Modeling and Reliability Considerations," 2017.

[2] D. Kumar, F. Zare, and A. Ghosh, "DC Microgrid Technology: System Architectures, AC Grid Interfaces, Grounding Schemes, Power Quality, Communication Networks, Applications and Standardizations Aspects," IEEE Access, pp. 1-1, 2017.

[3] L. Meng et al., "Review on Control of DC Microgrids and Multiple Microgrid Clusters Lexuan," IEEE J. Emerg. Sel. Top. Power Electron., vol. 6777, no. c, pp. 1-1, 2017.

[4] T. Dragicevic, X. Lu, J. Vasquez, and J. Guerrero, "DC MicrogridsPart I: A Review of Control Strategies and Stabilization Techniques," IEEE Trans. Power Electron., pp. 4876-4891, 2016.

[5] T. Dragicevic, X. Lu, J. C. Vasquez, and J. M. Guerrero, "DC Microgrids-Part II: A Review of Power Architectures, Applications, and Standardization Issues," IEEE Trans. Power Electron., vol. 31, no. 5, pp. 3528-3549, May 2016.

[6] D. Chen, Y. Xu, and A. Q. Huang, "Integration of DC Microgrids as Virtual Synchronous Machines into the AC Grid," IEEE Trans. Ind. Electron., pp. 1-1, 2017.

[7] J. Yang et al., "Decentralised control method for DC microgrids with improved current sharing accuracy," IET Gener. Transm. Distrib., vol. 11, no. 3, pp. 696-706, 2017.

[8] Y. Mi, H. Zhang, S. Member, Y. Fu, and C. Wang, "Intelligent Power Sharing of DC Isolated Microgrid Based on Fuzzy Sliding Mode Droop Control," vol. 3053, no. c, 2017.

[9] P. Prabhakaran, Y. Goyal, and V. Agarwal, "Novel Nonlinear Droop Control Techniques to Overcome the Load Sharing and Voltage Regulation Issues in DC Microgrid," IEEE Trans. Power Electron., pp. 1-1, 2017.

[10] Y. Wang et al., "Aggregated Energy Storage for Power System Frequency Control: A Finite-Time Consensus Approach," IEEE Trans. Smart Grid, pp. 1-1, 2018.

[11] J. C. Hernández, P. G. Bueno, and F. Sanchez-Sutil, "Enhanced utility-scale photovoltaic units with frequency support functions and dynamic grid support for transmission systems," IET Renew. Power Gener., vol. 11, no. 3, pp. 361-372, Feb. 2017.

[12] National Grid, "Mandatory Frequency Response." [Online]. Available:

https://www.nationalgrid.com/sites/default/files/documents/Mandat ory Frequency Response Guide v1.1.pdf.

[13] F. Teng, M. Aunedi, D. Pudjianto, and G. Strbac, "Benefits of Demand-Side Response in Providing Frequency Response Service in the Future GB Power System," Front. Energy Res., vol. 3, no. April, 2015.

[14] National Grid, "Testing guidance for providers of enhanced frequency response balancing service," 2017.

[15] A. F. Hoke, M. Shirazi, S. Chakraborty, E. Muljadi, and D. Maksimovic, "Rapid Active Power Control of Photovoltaic Systems for Grid Frequency Support," IEEE J. Emerg. Sel. Top. Power
Electron., vol. 5, no. 3, pp. 1154-1163, Sep. 2017.

[16] X. Lyu, Z. Xu, J. Zhao, and K. P. Wong, "Advanced frequency support strategy of photovoltaic system considering changing working conditions," IET Gener. Transm. Distrib., vol. 12, no. 2, pp. 363-370, 2018.

[17] Y. Fu, Y. Wang, and X. Zhang, "Integrated wind turbine controller with virtual inertia and primary frequency responses for grid dynamic frequency support," pp. 1129-1137, 2017.

[18] B. Gundogdu, S. Nejad, D. T. Gladwin, and D. A. Stone, "A battery energy management strategy for UK enhanced frequency response," in 2017 IEEE 26th International Symposium on Industrial Electronics (ISIE), 2017, pp. 26-31.

[19] Y. Wang et al., "Aggregated Energy Storage for Power System Frequency Control: A Finite - Time Consensus Approach," IEEE Trans. Smart Grid, vol. 3053, no. c, pp. 1-12, 2017.

[20] Di. Chakravorty, B. Chaudhuri, and S. Y. R. Hui, "Rapid Frequency Response from Smart Loads in Great Britain Power System," IEEE Trans. Smart Grid, vol. 8, no. 5, pp. 2160-2169, 2017.

[21] P. Nuutinen, A. Lana, and T. Hakala, "Lvdc Rules - Technical Specifications for Public Lvdc Distribution Network," Cired 2017, no. June, pp. 12-15, 2017

[22] A. Emhemed and G. Burt, "Protection analysis for plant rating and power quality issues in LVDC distribution power systems," in 2015 IEEE Power \& Energy Society General Meeting, 2015, pp. 1-5.

[23] M. V. Gururaj and N. P. Padhy, "A Novel Decentralized Coordinated Voltage Control Scheme for Distribution System With DC Microgrid," IEEE Trans. Ind. Informatics, vol. 3203, no. c, pp. 1-1, 2017.

[24] E. Rikos, "Photovoltaic Systems Modelling for Simulation Purposes What is the importance of simulating PV systems ?," no. 654113.

[25] Y. Gu, W. Li, and X. He, "Frequency-coordinating virtual impedance for autonomous power management of DC microgrid," IEEE Trans. Power Electron., vol. 30, no. 4, pp. 2328-2337, 2015. 